Supporting Information for

\title{
Deciphering the 3D Microstructures of a Doubly Charged Homopolymer through a Complementary Correlation of Monomer Crystallography and Polymer Powder X-ray Diffraction
}

\author{
Xi Chen, ${ }^{1}$ Glenn A. Spiering, ${ }^{1}$ Carla Slebodnick, ${ }^{1}$ Timothy E. Long, ${ }^{1}$ and Robert B. Moore ${ }^{1, *}$ \\ ${ }^{1}$ Department of Chemistry, Macromolecules Innovation Institute (MII), \\ Virginia Tech, Blacksburg, 24061, United States \\ *Email: rbmoore3@vt.edu
}

\section{Experimental Section}

\section{Materials}

2-(Dodecylthiocarbonothioylthio)-2-methylpropionic acid (DDMAT) was purchased from Sigma-Aldrich and used as received. 4-(dimethylamino) pyridine (DMAP, Sigma-Aldrich, $\geq 99 \%$ ), a, $\alpha^{\prime}$-Azobis(isobutyronitrile) (AIBN, Fluka, 99\%), dicyclohexylcarbodiimide (DCC, SigmaAldrich, 99\%), 1,4-diazabicyclo[2.2.2]octane (DABCO, Sigma-Aldrich, 99\%), 1bromooctadecane (Sigma-Aldrich, 99\%), 1,6-hexanediamine (Sigma-Aldrich, 99\%), 2,6-di-tertbutyl-4-methylphenol (BHT, Sigma-Aldrich, 99\%) were used without further purification. $\alpha, \alpha^{\prime}-$ Azobis(isobutyronitrile) (AIBN, 99\%) was obtained from Fluka and recrystallized twice from methanol before use. Ethyl acetate (Spectrum, HPLC grade), N, N-dimethylformamide (DMF, Spectrum, HPLC grade), methanol (MeOH, Fisher Chemical, HPLC grade), chloroform (Fisher Chemical, HPLC grade), ethylene glycol (EG, TCI, 99.5\%), and N-methyl-2-pyrrolidone (NMP, Sigma-Aldrich, HPLC grade) were used as purchased.

\section{Analytical Methods}


${ }^{1} \mathrm{H}$ nuclear magnetic resonance (NMR) spectroscopy were performed using an Agilent U4-DD2 $400 \mathrm{~Hz}$ spectrometer at $23{ }^{\circ} \mathrm{C}$. The hompolymer was dissolved a mixture of $\mathrm{CD}_{3} \mathrm{OD}$ and $\mathrm{CDCl}_{3}$ $(1: 1 \mathrm{v} / \mathrm{v})$. Differential scanning calorimetry (DSC) was conducted on a TA Instruments DSC Q2000 from -80 to $140{ }^{\circ} \mathrm{C}$ under $\mathrm{N}_{2}$ purge using a heat/cool/heat cycle at a heating or cooling rate of $10^{\circ} \mathrm{C} / \mathrm{min}$.

Small angle X-ray scattering (SAXS) and powder X-ray diffraction (PXRD) experiments were performed using a Rigaku S-Max 30003 pinhole SAXS system, equipped with a rotating anode emitting X-ray with a wavelength of $0.154 \mathrm{~nm}(\mathrm{Cu} \mathrm{K \alpha})$. The sample-to-detector distance was 1005 mm for SAXS and $195 \mathrm{~mm}$ for PXRD, and the q-range was calibrated using a silver behenate standard. Two-dimensional SAXS patterns were obtained using a 2D multiwire, proportional counting, gas-filled detector, with an exposure time of $2 \mathrm{~h}$. Two dimensional PXRD diffraction patterns were obtained using an image plate with an exposure time of $1 \mathrm{~h}$. The SAXS data were corrected for sample thickness, transmission, and background, and were put on an absolute scale by correction using a glassy carbon standard from the Advanced Photon Source (APS). The PXRD data were corrected for background. The SAXS/PXRD profiles were vertically shifted to facilitate a comparison of peak positions. All the SAXS and PXRD data were analyzed using the SAXSGUI software package to obtain radially integrated SAXS and PXRD intensity versus the scattering vector $\mathrm{q}(\mathrm{SAXS})$ or $2 \theta(\mathrm{PXRD})$ respectively, where $\mathrm{q}=(4 \pi / \lambda) \sin (\theta), \theta$ is one half of the scattering angle and $\lambda$ is the $\mathrm{X}$-ray wavelength.

Single crystal X-ray diffraction (SCXRD) and variable temperature powder X-ray diffraction (PXRD) studies were performed on a Rigaku Oxford Diffraction Synergy-S single-crystal diffractometer operating with $\mathrm{CuK} \alpha$ radiation and equipped with a HyPix6000HE area detector and an Oxford Cryostream 800 Plus temperature controller. The program CrysAlisPro ${ }^{1}$ was used 
for data collection, to process the single crystal data, and to extract the powder patterns from the raw image.

For the single crystal of $\mathrm{VBDC}_{18} \mathrm{BrCl}$ monomer, the Laue symmetry and systematic absences were consistent with the monoclinic space group $P 2{ }_{1} / c$. The structure was solved using SHELXT ${ }^{2}$ and refined using SHELXL ${ }^{3}$ via Olex $2 .{ }^{4}$ The final structure model included the organic dication, two halide anions, and a water solvate. A 2-position disorder model was used for the 4-vinylbenzyl group, with relative occupancies that refined to $0.677(11)$ and $0.323(11)$. The anisotropic displacement parameters of the atoms of the disordered group were restrained with RIGU. In addition, EADP constraints were used for $\mathrm{C} 9 \mathrm{~A} / \mathrm{C} 9 \mathrm{~B}$ and $\mathrm{C} 13 \mathrm{~A} / \mathrm{C} 13 \mathrm{~B}$. $\mathrm{A} \mathrm{Cl} / \mathrm{Br}$ composition disorder model was used for the two halide ions. The positions and anisotropic displacement parameters for ions occupying the same site were constrained to be equal using EXYZ and EADP. The relative occupancies for $\mathrm{Br} 1 / \mathrm{Cl} 1$ and $\mathrm{Br} / \mathrm{Cl} 2$ refined to 0.594(4)/ 0.406(4) and 0.647(4)/ 0.353(4), respectively. A large residual electron density peak $\sim 3.2-3.3 \AA$ from the halide ions was assumed to be water to give the overall chemical formula $\left[\mathrm{C}_{33} \mathrm{H}_{58} \mathrm{~N}_{2}\right] \mathrm{Br}_{1.24} \mathrm{Cl}_{0.76} \cdot \mathrm{H}_{2} \mathrm{O}$. The final refinement model involved anisotropic displacement parameters for all non-hydrogen atoms. The H-atom positions of the water molecule were located from the difference electron density map and refined independently. The isotropic displacement parameters of the H-atoms of water were constrained to $1.2 \mathrm{U}_{\mathrm{eq}}$ of the attached $\mathrm{O}$-atom.

Variable temperature PXRD studies on the single-crystal instrument were performed in DebyeScherrer configuration. Sample preparation of the monomer consisted of grinding and packing the as synthesized monomer powder into a $0.5 \mathrm{~mm}$ quartz capillary (Charles Supper) and sealing the open end of the capillary with super glue. For the homopolymer, a small fragment was adhered to a Nylon mounting loop (Hampton Research) with a small amount of oil. This sample was centered 
on the goniometer so that the X-ray hit just above the Nylon mount to avoid scattering from the Nylon or oil. Each data collection consisted of a 360 deg phi-scan over 5 min.

\section{Single Crystal Growth of DABCO Salt-Containing Monomer (VBDC $18 B r C l)$}

$\mathrm{VBDC}_{18} \mathrm{BrCl}$ monomer was prepared following previous literature in our group. ${ }^{5}$ Briefly, DABCO (10.0 g, $89.2 \mathrm{mmol}), 1$-bromooctadecane (26.7 g, $80.2 \mathrm{mmol})$, and ethyl acetate (200 mL) were charged into a $500 \mathrm{~mL}$ round-bottom flask equipped with a magnetic stir bar. The reaction mixture was stirred at room temperature for $24 \mathrm{~h}$. A white precipitate was filtered, washed with ethyl acetate, and dried in vacuo at room temperature overnight. The alkylated DABCO intermediate (24.8 g, $55.7 \mathrm{mmol}), 4 \mathrm{VBCl}(9.4 \mathrm{~g}, 61.3 \mathrm{mmol})$, and BHT (120 mg) were dissolved in $100 \mathrm{~mL}$ of $\mathrm{CHCl}_{3}$ and stirred at room temperature for $12 \mathrm{~h}$. A white product precipitated from the solution, collected, and washed with $\mathrm{CHCl}_{3}$. The resulting solids were dried under reduced pressure at room temperature for $12 \mathrm{~h}$ to afford $25.6 \mathrm{~g}$ of $\mathrm{DABCO}$ monomer $\left(\mathrm{VBDC}{ }_{18} \mathrm{BrCl}\right)$ with a yield of $78 \% .20 \mathrm{mg}$ as-prepared monomer powder was dissolved in $1 \mathrm{~mL}$ methanol and the solution was passed through cotton to remove dust particles. A vial containing the filtered solution was placed in a closed reservoir filled with a large amount of ether. The closed system sat at room temperature undisturbed to allow crystal growth through vapor diffusion. Colorless plates were obtained after $\sim 3$ weeks and the single crystal chosen for analysis had dimension $0.03 \mathrm{~mm} \times 0.11$ $\mathrm{mm} \times 0.20 \mathrm{~mm}$.

\section{Synthesis of VBDC18BrCl Homopolymer Poly (VBDC18BrCl)}

A difunctional chain transfer agent (di-CTA), 1,6-bis(2-(dodecylthiocarbono-thioylthio)-2methylpropionic acid)-hexane diamide (diDDMAT-NH$)$ was afforded using an adapted literature

procedure. $^{6}$ To a $50 \mathrm{~mL}$ Schlenk flask, $2 \mathrm{~g}$ (3.34 mmol) $\mathrm{VBDC}_{18} \mathrm{BrCl}, 20.79 \mathrm{mg}(0.026 \mathrm{mmol})$ 
diDDMAT-NH 2 (monomer/CTA=130), $0.42 \mathrm{mg}(0.0026 \mathrm{mmol})$ AIBN (CTA/I=10) were added, the solids were dissolved in $7 \mathrm{~mL} \mathrm{DMF} / \mathrm{MeOH})$ cosolvent (3:4, wt/wt, $20 \mathrm{wt} \%$ solids). After four freeze-pump-thaw cycles followed by backfilling with argon, the reaction mixture was stirred at $70{ }^{\circ} \mathrm{C}$ for $24 \mathrm{~h}$ with a conversion of $78 \%$ (Figure S1). The reaction solution was purified through dialysis in a $\mathrm{MeOH} / \mathrm{CHCl}_{3}$ cosolvent for $3 \mathrm{~d}$ and the resulting polymer was dried at $50{ }^{\circ} \mathrm{C}$ to yield brittle yellow solids. ${ }^{1} \mathrm{H} \mathrm{NMR}$ in a $\mathrm{CDCl}_{3} / \mathrm{CD}_{3} \mathrm{OD}$ mixture confirmed the polymer chemical structure (Figure S2).

\section{Polymer Solvent Annealing Procedures}

Poly $\left(\mathrm{VBDC}_{18} \mathrm{BrCl}\right)$ homopolymer was dissolved $5 \mathrm{wt} \%$ in ethylene glycol/NMP (2:3, wt/wt) cosolvent and the solution was added into a Teflon mold. The mold was covered with a petri dish and placed at $80^{\circ} \mathrm{C}$ to allow slow solvent evaporation. Upon solvent removal $(\sim 3-5 \mathrm{~d})$, the polymer was further dried under reduced pressure at room temperature for $24 \mathrm{~h}$ and was then annealed at $80{ }^{\circ} \mathrm{C}$ for $24 \mathrm{~h}$. After slow cooling to room temperature, the mold was stored in a desiccator prior to characterization. A controlled poly $\left(\mathrm{VBDC}_{18} \mathrm{BrCl}\right)$ sample was cast from a $\mathrm{MeOH} / \mathrm{CHCl}_{3}$ mixture at room temperature and annealed following the identical procedures. 


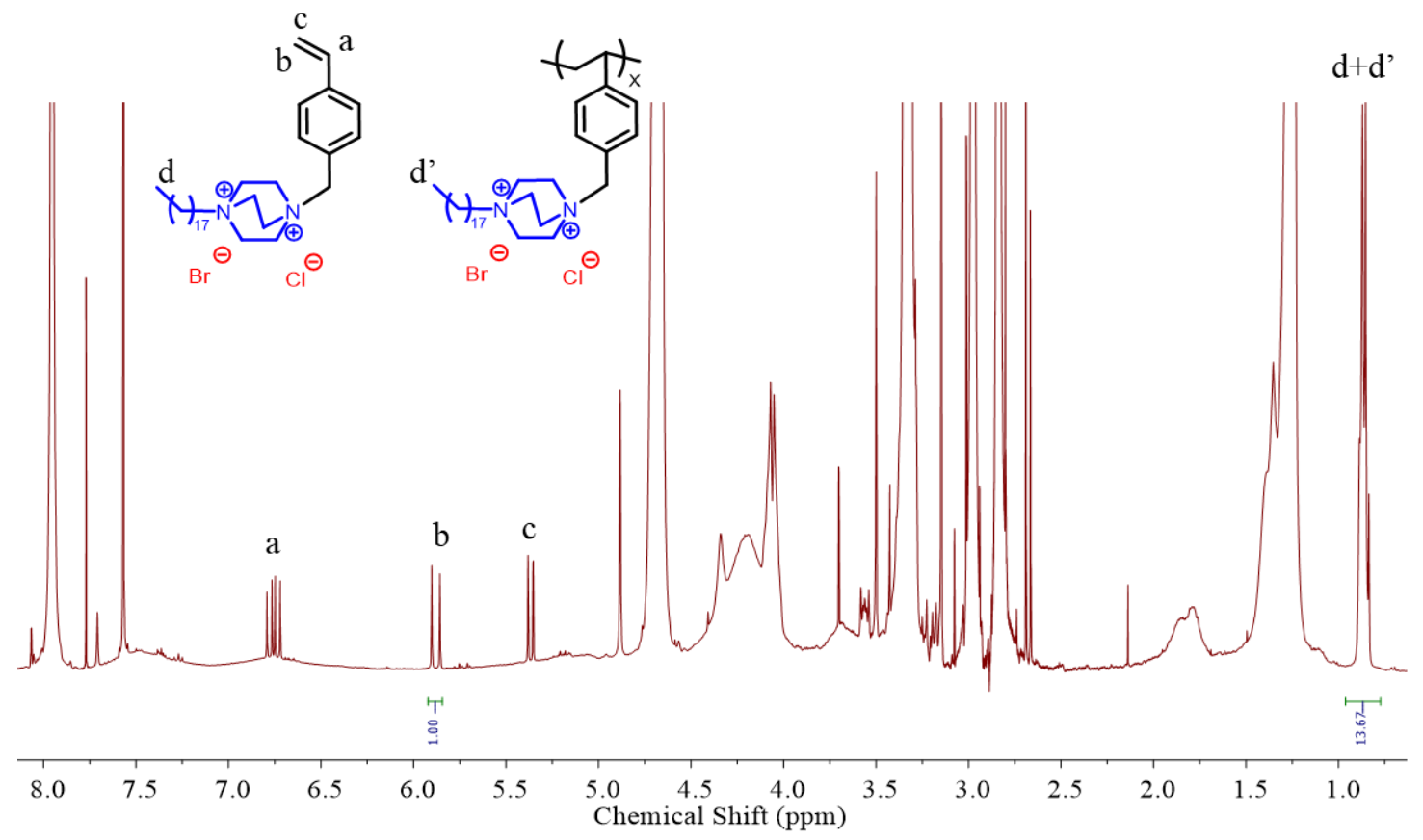

Figure S1. ${ }^{1} \mathrm{H}$ NMR of poly $\left(\mathrm{VBDC}_{18} \mathrm{BrCl}\right)$ homopolymer reaction mixture for conversion calculation. ${ }^{1} \mathrm{H} \mathrm{NMR}$ is performed in a $\mathrm{CDCl}_{3} / \mathrm{CD}_{3} \mathrm{OD}$ mixture

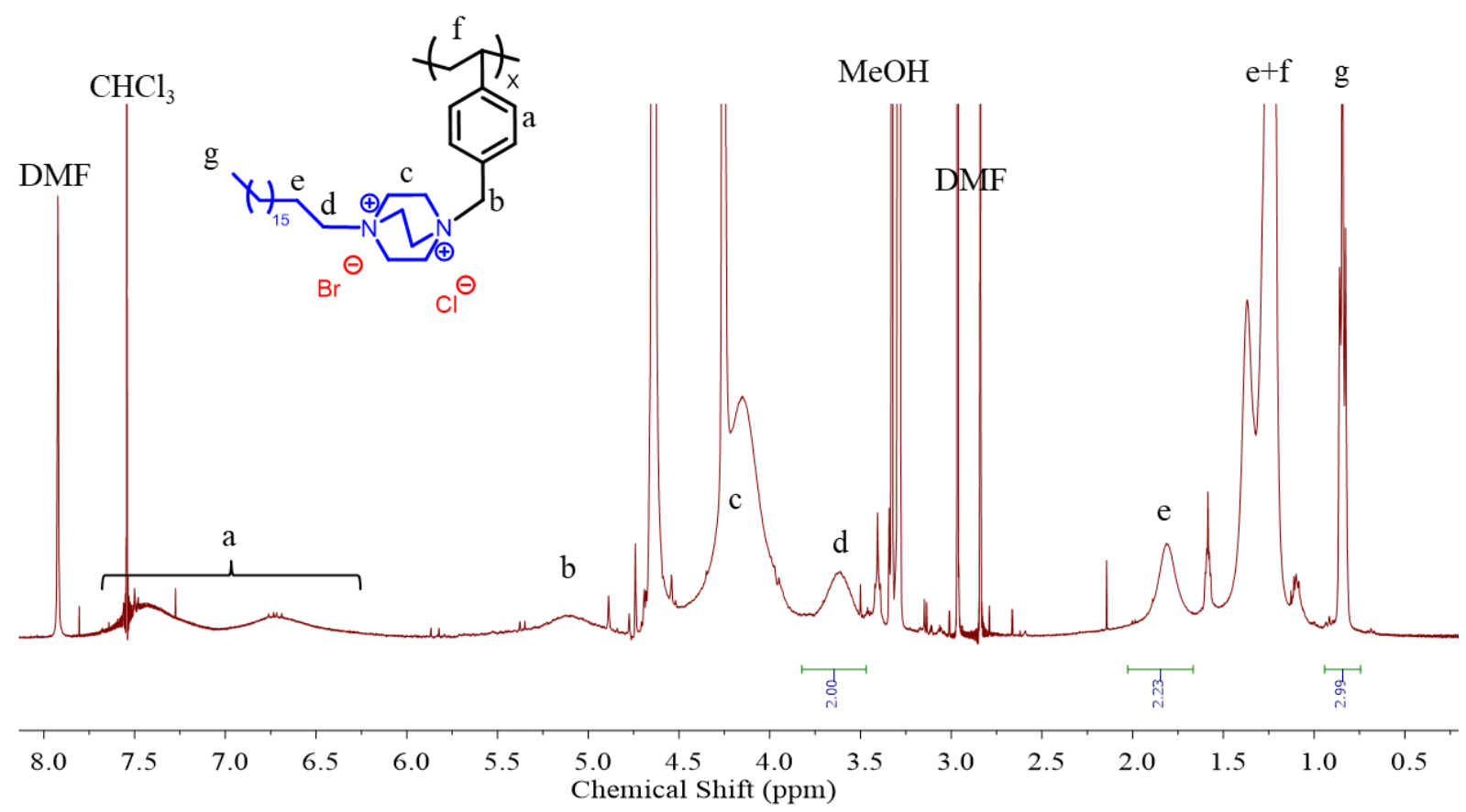

Figure S2. ${ }^{1} \mathrm{H}$ NMR of purified poly $\left(\mathrm{VBDC}_{18} \mathrm{BrCl}\right) .{ }^{1} \mathrm{H} \mathrm{NMR}$ is performed in a $\mathrm{CDCl}_{3} / \mathrm{CD}_{3} \mathrm{OD}$ mixture 


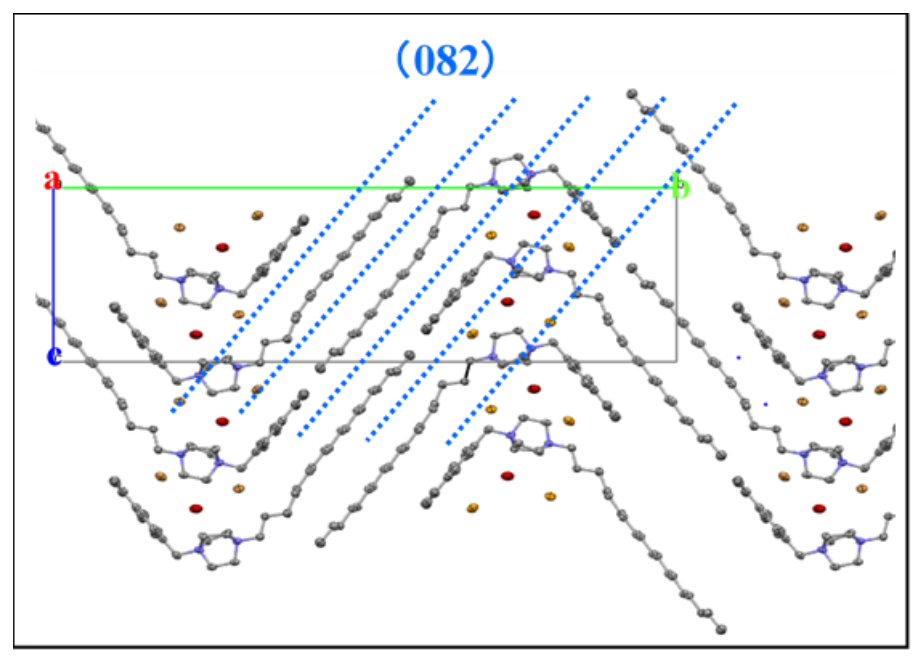

Figure S3. Packing diagram of the single-crystal X-ray structure of the monomer with the interplanar spacing of (082) planes indicating the alkyl-alkyl correlation distance. The crystallographic axes are also shown on each image, where $a$-axis $=$ red, $b$-axis $=$ green, and $c$-axis $=$ blue

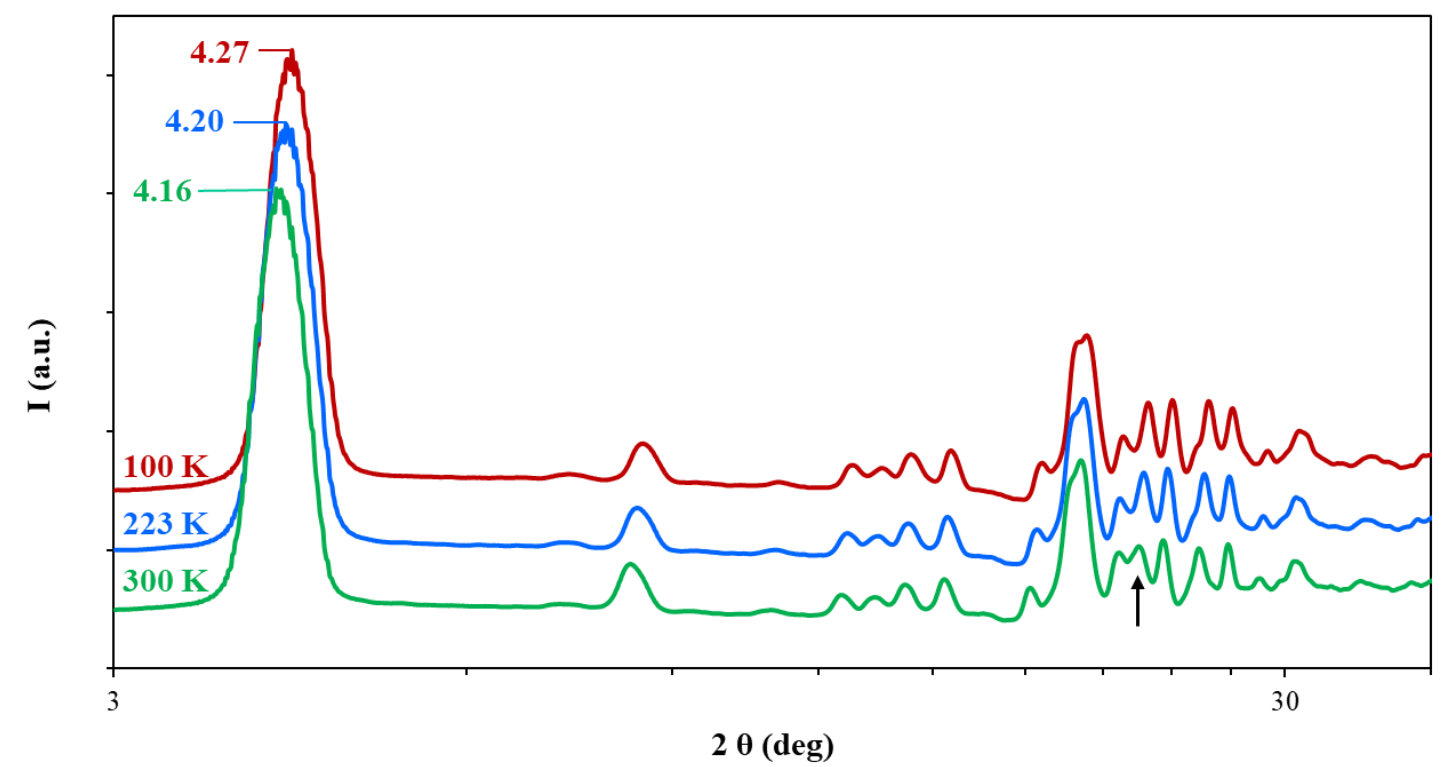

Figure S4. PXRD of the $\mathrm{VBDC}_{18} \mathrm{BrCl}$ monomer at varied temperatures, the black arrow indicates a decrease of (082) intensity at $300 \mathrm{~K}$. 


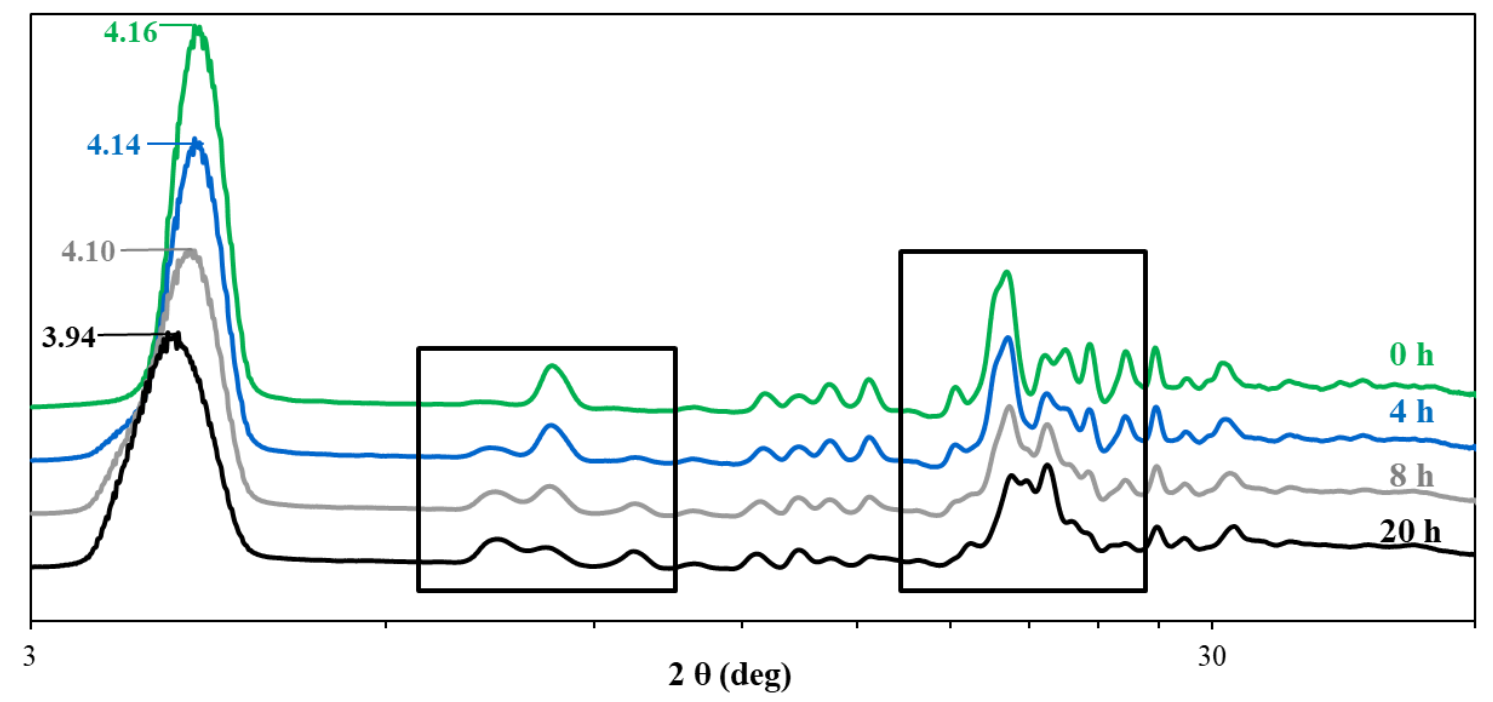

Figure S5. The $\mathrm{VBDC}_{18} \mathrm{BrCl}$ monomer displays radiation decay at $300 \mathrm{~K}$ with time during PXRD analysis
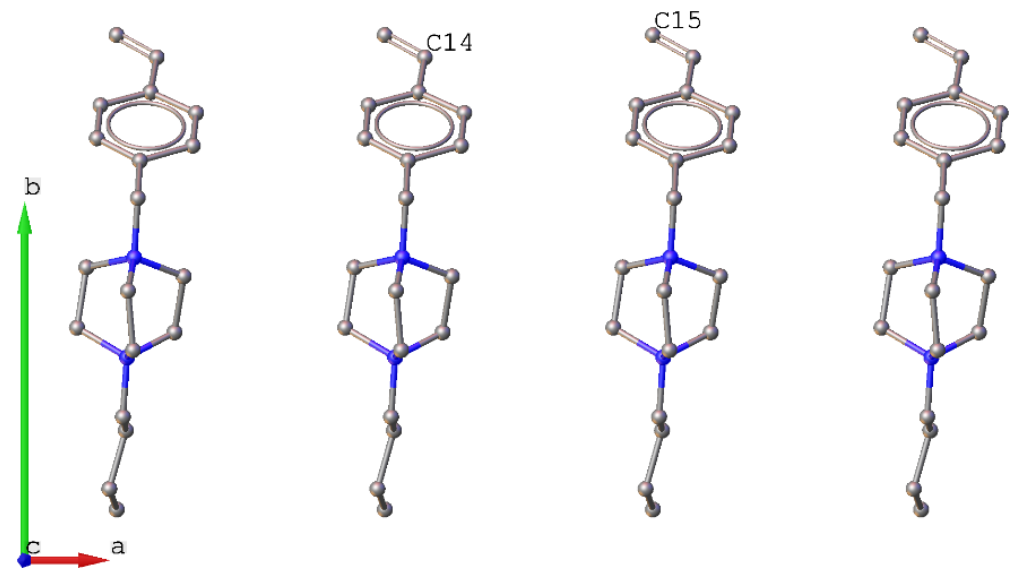

Figure S6. Partial structure of $\mathrm{VBDC}_{18} \mathrm{Br}_{1.24} \mathrm{Cl}_{0.76} \cdot \mathrm{H}_{2} \mathrm{O}$ viewed down the crystallographic $c$-axis depicting the packing of the molecule fragment comprising the major disorder conformation of the styrene, the DABCO, and the C16-C19. The vinyl groups of the styrene are aligned perfectly for polymerization to occur parallel to the crystallographic $a$-axis. The interatomic $\mathrm{C} 14 \ldots \mathrm{C} 15$ distance is 5.831(10) $\AA$. 


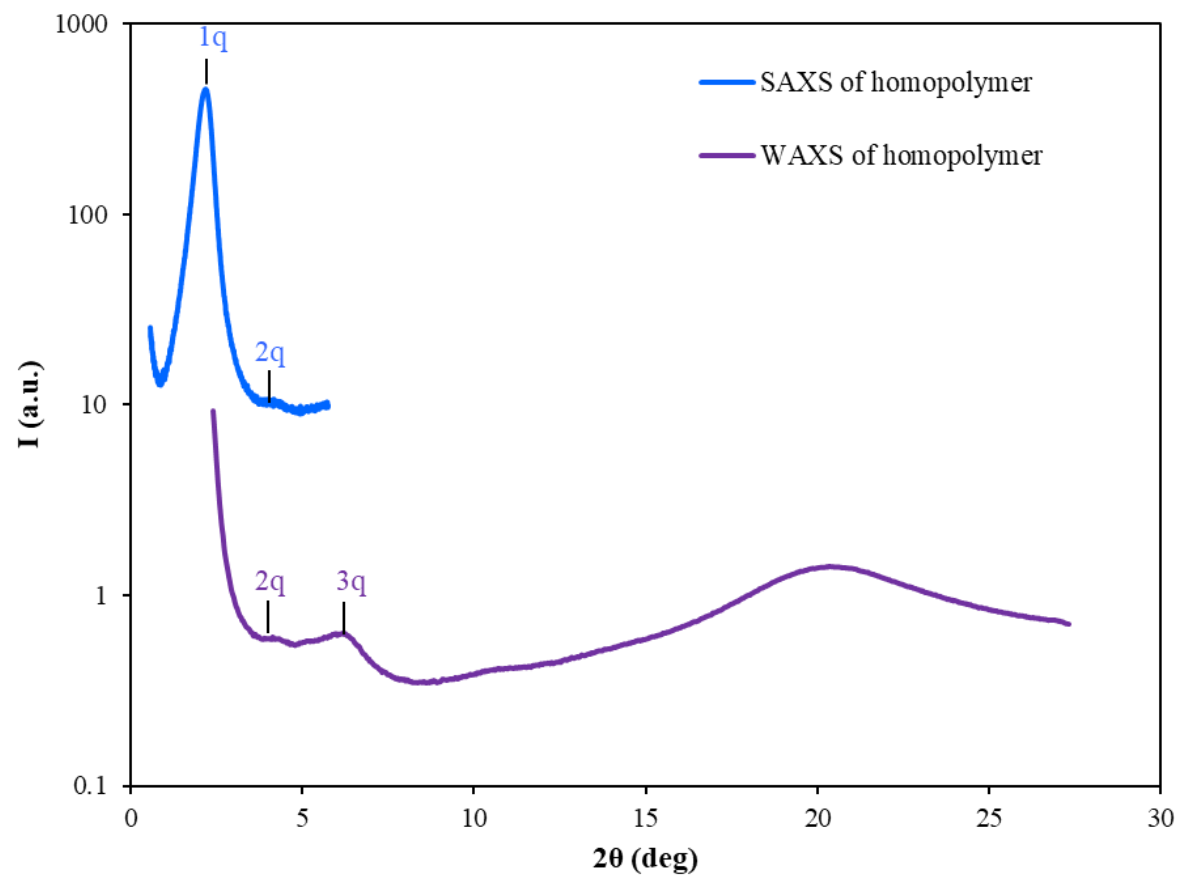

Figure S7. X-ray scattering of poly $\left(\mathrm{VBDC}_{18} \mathrm{BrCl}\right)$ casted from $\mathrm{MeOH} / \mathrm{CHCl}_{3}$ cosolvent at $300 \mathrm{~K}$

\section{References}

(1) CrysAlisPro Software System, v1.171.40.53, Rigaku Oxford Diffraction; Rigaku

Corporation: Oxford, UK, 2019.

(2) Sheldrick, G. M. SHELXT - Integrated Space-Group and Crystal Structure

Determination. Acta Cryst 2015, A71, 3-8.

(3) Sheldrick, G. M. Crystal Structure Refinement with SHELXL. Acta Cryst 2015, C71, 3-8.

(4) Dolomanov, O.V.; Bourhis, L. J.; Gildea, R. J.; Howard, J. A. K.; Puschmann, H. OLEX2:

A Complete Structure Solution, Refinement and Analysis Program. J. Appl. Cryst. 2009, 42, 339-341.

(5) Chen, X.; Talley, S. J.; Haag, J. V.; Spiering, G. A.; Liu, B.; Drummey, K. J.; Murayama, M.; Moore, R. B.; Long, T. E. Doubly Charged ABA Triblock Copolymers: 
Thermomechanically Robust Physical Network and Hierarchical Microstructures.

Macromolecules 2019, 52, 9168-9176.

(6) Allen, M. H.; Hemp, S. T.; Zhang, M.; Zhang, M.; Smith, A. E.; Moore, R. B.; Long, T. E. Synthesis and Characterization of 4-Vinylimidazole ABA Triblock Copolymers Utilizing a Difunctional RAFT Chain Transfer Agent. Polym. Chem., 2013, 4, 2333-2341. 